\title{
Simultaneous Bright Field and Dark Field STEM-IN-SEM Imaging of Polymer Nanocomposites
}

\author{
B. Patel, M. Watanabe
}

Dept. of Material Science and Engineering, Lehigh University, Bethlehem, PA 18015

Scanning transmission electron microscopy in scanning electron microscopy (STEM-IN-SEM) [e.g. 1] is a convenient technique for polymer characterization. Utilizing the lower accelerating voltages, larger field of view and, exclusion of post-specimen projection lens in an SEM; STEM-IN-SEM has shown results comparable to transmission electron microscopy (TEM) observation of polymer morphology [2]. Various specimen-holder geometries and detector arrangements have been used for STEM-IN-SEM imaging [e.g. 3, 4]. Here we present the latest updates in our development of simultaneous bright field (BF) and dark field (DF) STEM-IN-SEM imaging.

Figure 1 shows the updated specimen holder. A washer is located at the top of the holder, under which a TEM-like thin specimen can be mounted in a recess. An electron mirror coated with heavy metal is positioned at the middle of the holder to reflect highly scattered electrons for DF imaging. In contrast, the holder body and the overhead washer are coated with graphite to minimize undesirable electron emission from them. Since our initial prototype [5] the DF signal acquisition has been optimized by improving the electron mirror design. Now the electron mirror is made of a flat-polished copper substrate that has been coated with a thick layer of gold and bent preferentially toward the DF detector. It should be noted that this particular holder developed in this study has been designed to fit into a Hitachi S-4300SE/N instrument at Lehigh University. However, the design concept should be applicable to any existing SEM instrument without major modification.

Figure 2 shows a schematic representation of the set up for BF and DF STEM-IN-SEM imaging. An incident electron beam scans the specimen. The interaction between the incident electron beam and the specimen results in the transmission of forward-scattered electrons. Low-angle scattered electrons pass through an aperture opening in the middle of the electron mirror and are collected by a STEM detector positioned directly under the specimen for a BF image. High-angle scattered electrons are reflected on the electron mirror and are collected by an off-axis yttrium-aluminum garnet (YAG) detector, which is usually used for BSE imaging. This configuration excludes the direct electron beam in image formation and thus results in a DF image.

Figure 3 shows a pair of BF and DF images from an ultramicrotomed and $\mathrm{OsO}_{4}$-stained epoxy-based nanocomposite filled with 25 parts per hundred resin E20-grade copolymer of polystyrene, 1,4polybutadiene and syndiotactic poly (methyl methacrylate) (25 phr E20 SBM). The BF image (left) exhibits mass-thickness contrast. In the $\mathrm{BF}$ image, darker regions represent the preferentially $\mathrm{OsO}_{4}$ stained polybutadiene (PB) phase which surrounds an inner polystyrene (PS) core. Surrounding the PB phase is a poly (methyl methacrylate) (PMMA) layer that is difficult to distinguish from the epoxy matrix given their high mutual miscibility. The DF image (right) seems more intuitive to interpret because the contrast mainly relates to local atomic number ( $Z$ ). In the DF image, the brightest regions are $\mathrm{OsO}_{4}$-stained PB while the PS appears as a different gray scale and the PMMA phase and epoxy matrix are the darkest regions. 
Figure 4 shows a BF image collected on a JEOL-2000FX TEM from an ultramicrotomed and $\mathrm{OsO}_{4}$ stained epoxy-based nanocomposite filled with 25 phr E20 SBM. The BF image exhibits massthickness contrast with comparable contrast to the BF STEM-IN-SEM image (Figure 3 (left)). However the BF image show signs of beam damage (brightest regions) even with a spread beam and signs of over-staining (darkest regions) which make it difficult to detect the PS core.

Simultaneous BF and DF STEM-IN-SEM imaging is useful for measuring particle size and distribution from a relatively large field of view. Further enhancements of imaging conditions and sample preparation are actively ongoing to allow for the imaging of unstained polyme nanocomposites, which is one of the long-term dreams for observation of carbon-based materials in SEM or TEM.

\section{References}

[1] D. C. Joy and D. M. Maher, Scan. Elect. Microsc. (IIT Research Institute, Chicago, 1976) 361.

[2] O. Guise et al., Polymer 52 (2011) 1278.

[3] W. E. Vanderlinde and V. W. Ballarotto, Proceedings of $30^{\text {th }}$ ISTFA (Boston, 2004) 1.

[4] A. Bogner et al., Micron 38 (2007) 390.

[5] B. Patel and M. Watanabe, Microscopy \& Microanalysis 18 Suppl. 2 (2012) 1236.

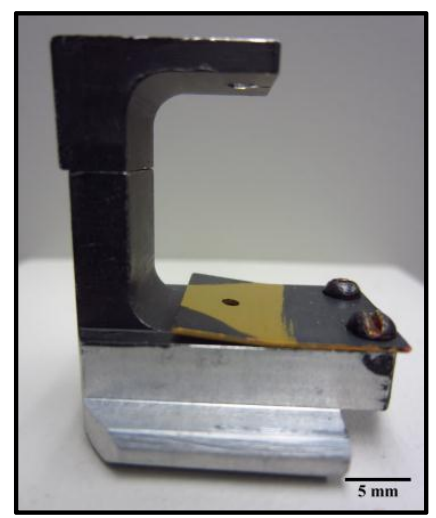

FIG. 1: Updated simultanous BF and DF STEM-IN-SEM specimen holder for Hitachi S-4300SE/N.

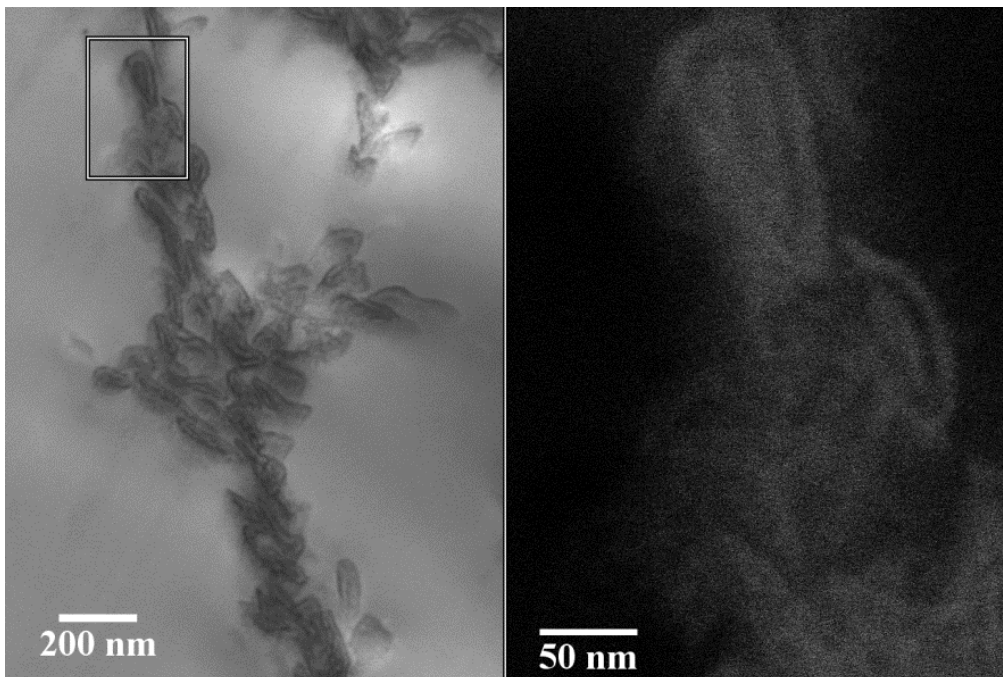

FIG. 3: BF (left) and DF (right) images simultaneously acquired from ultramicrotomed and $\mathrm{OsO}_{4}$-stained epoxy nanocomposite filled with 25 phr E20 SBM by STEM-in-SEM at $30 \mathrm{kV}$.

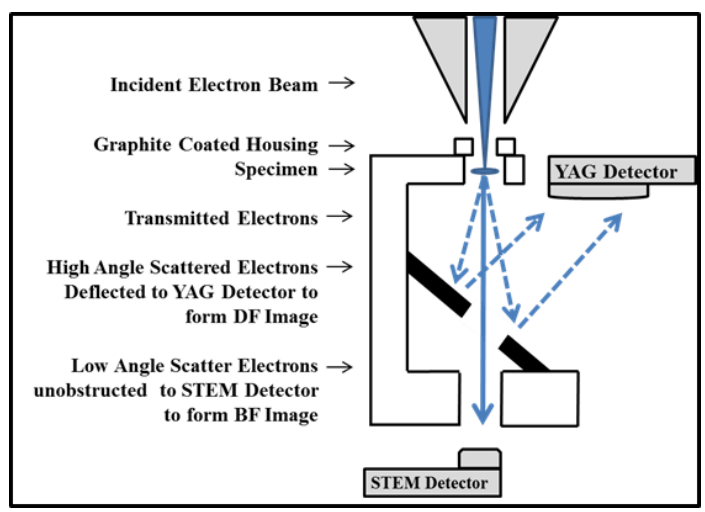

FIG. 2: A schematic diagram of BF and DF STEM-IN-SEM imaging.

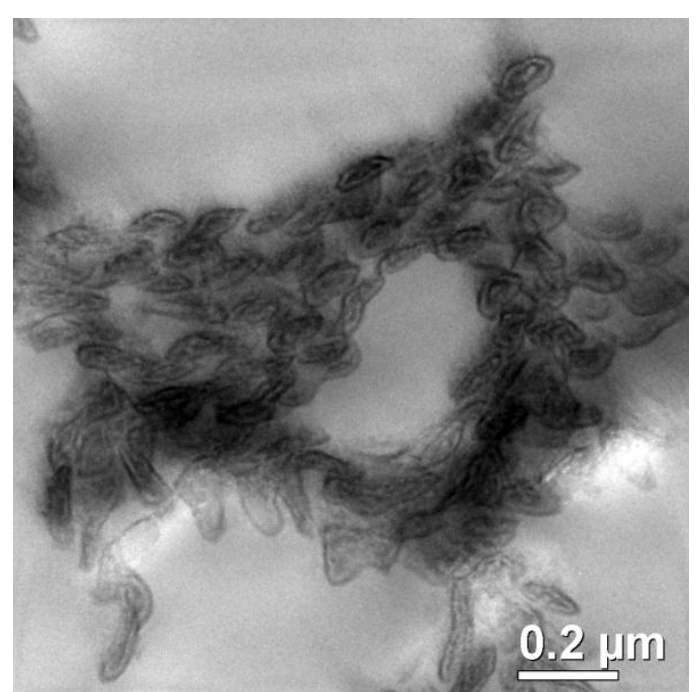

FIG. 4: BF image acquired from ultramicrotomed and $\mathrm{OsO}_{4}$-stained epoxy nanocomposite filled with $25 \mathrm{phr}$ E20 SBM by TEM at $200 \mathrm{kV}$. 